\title{
Pazopanib for severe bleeding and transfusion-dependent anemia in hereditary hemorrhagic telangiectasia
}

\author{
Joseph G. Parambil ${ }^{1}$. James R. Gossage ${ }^{2} \cdot$ Keith R. McCrae $^{3} \cdot$ Troy D. Woodard $^{4} \cdot$ K. V. Narayanan Menon ${ }^{5}$ \\ Kasi L. Timmerman ${ }^{1} \cdot$ Douglas P. Pederson $^{1} \cdot$ Dennis L. Sprecher ${ }^{6} \cdot$ Hanny AI-Samkari ${ }^{7} \mathbb{C}$
}

Received: 22 March 2021 / Accepted: 7 July 2021 / Published online: 22 July 2021

(c) The Author(s), under exclusive licence to Springer Nature B.V. 2021

\begin{abstract}
Hereditary hemorrhagic telangiectasia (HHT) is a rare angiogenic disorder causing chronic gastrointestinal bleeding, epistaxis, and severe anemia. Pazopanib is an oral multi-kinase angiogenesis inhibitor with promise to treat bleeding in HHT. We analyzed outcomes of HHT patients with the most severe bleeding causing RBC transfusion dependence treated on a predefined institutional pazopanib treatment pathway (with data collected retrospectively). The primary endpoint was achievement of transfusion independence. Secondary endpoints included hemoglobin, epistaxis severity score, RBC transfusion and iron infusion requirements, number of local hemostatic procedures, ferritin and transferrin saturation, compared using paired and repeated measures mean tests. Thirteen transfusion-dependent HHT patients received pazopanib [median (range) dose 150 (25-300) mg daily)] for a median of 22 months. All patients achieved transfusion independence. Compared with pretreatment, pazopanib increased mean hemoglobin by $4.8(95 \% \mathrm{CI}, 3.6-5.9) \mathrm{g} / \mathrm{dL}(7.8 \mathrm{vs} .12 .7 \mathrm{~g} / \mathrm{dL}, P<0.0001)$ and decreased mean epistaxis severity score by $4.77(3.11-6.44)$ points (7.20 vs. 2.43 points, $P<0.0001)$ after 12 months of treatment. Compared with 3 months of pretreatment, RBC transfusions decreased by $93 \%$ (median of 16.0 vs. 0.0 units, $P<0.0001$ ) and elemental iron infusion decreased by $92 \%$ (median of $4500 \mathrm{vs.} 0 \mathrm{mg}, P=0.005$ ) during the first 3 months of treatment; improvements were maintained over time. Pazopanib was well-tolerated: hypertension, lymphocytopenia, and fatigue were the most common TEAEs. In conclusion, pazopanib was safe and effective to manage severe bleeding in HHT, liberating all patients from transfusion dependence and normalizing hematologic parameters at doses lower than used to treat malignancies. These findings require confirmation in a randomized trial.
\end{abstract}

Keywords Hereditary hemorrhagic telangiectasia $\cdot$ Osler-Weber-Rendu $\cdot$ HHT $\cdot$ Pazopanib $\cdot$ Angiogenesis $\cdot$ Bleeding · Epistaxis $\cdot$ Gastrointestinal bleeding $\cdot$ Anemia $\cdot$ Iron deficiency

\section{Introduction}

Hereditary hemorrhagic telangiectasia (HHT, Osler-WeberRendu disease) is an autosomal dominant rare bleeding disorder affecting 1 in 5000 persons, characterized by

Joseph G. Parambil

parambj@ccf.org

Hanny Al-Samkari

hal-samkari@mgh.harvard.edu

1 Cleveland Clinic, Respiratory Institute, 9500 Euclid Avenue A-90, Cleveland, OH 44195, USA

2 Division of Pulmonary, Critical Care and Sleep Medicine, Augusta University, Augusta, GA, USA

3 Cleveland Clinic, Taussig Cancer Institute, Cleveland, OH, USA arteriovenous malformations (AVMs) of both large and small vessels $[1,2]$. Causative mutations have been identified in six genes, all involved in the signaling cascades of angiogenesis, with most cases due to mutations of $E N G$ and ACVRL1 [3,4]. Severe, recurrent epistaxis is a near

4 Cleveland Clinic, Head and Neck Institute, Cleveland, OH, USA

5 Cleveland Clinic, Digestive Diseases Institute, Cleveland, $\mathrm{OH}$, USA

6 Cure HHT Foundation, Monkton, MD, USA

7 Division of Hematology, Massachusetts General Hospital, Zero Emerson Pl Suite 118, Office 112, Boston, MA 02114, USA 
universal finding and chronic gastrointestinal (GI) bleeding occurs. Both sources of unrelenting bleeding may result in severe iron deficiency anemia that is iron infusion and/or red blood cell (RBC) transfusion-dependent $[5,6]$. This chronic hemorrhage causes significant morbidity and increases mortality in HHT [7], as well as significantly reduces healthrelated quality of life for these patients $[8,9]$.

While there are no FDA-approved treatments for HHTassociated bleeding, advances in the understanding of disease pathobiology point to the role of excess vascular endothelial growth factor (VEGF), both locally and systemically $[10,11]$. This has generated intense interest in the use of anti-angiogenic therapies to manage HHT [12-14]. Systemic bevacizumab, a single-target anti-VEGF-A humanized monoclonal antibody, has been shown to improve clinical outcomes of high-output heart failure from liver vascular malformations and refractory bleeding in patients with HHT [15-18]. However, the uncertainty over optimal long-term dosing, requirement for intravenous administration, and potential risks of tachyphylaxis and deleterious side effects with extended use at oncologic doses $[15,19]$ have triggered a search for alternative agents.

One of these is oral pazopanib, a multi-target receptor tyrosine kinase inhibitor with potent anti-angiogenic properties, that has approval for the treatment of advanced renal cell carcinoma and certain subtypes of advanced soft tissue sarcoma at a starting dose of $800 \mathrm{mg}$ daily [20]. Prior study with a pazopanib analog was shown to inhibit AVM development in an HHT mouse model [21], and a case report and short pilot study have suggested promising activity in humans with HHT [22, 23].

Given the lack of an FDA-approved drug and the promise of pazopanib to treat bleeding in HHT, we developed an offlabel, predefined institutional treatment pathway to allow for structured pazopanib treatment and monitoring in patients with severe, transfusion-dependent HHT-associated bleeding and anemia. We hypothesized that pazopanib would be effective in reducing bleeding and thereby liberate patients from transfusion dependence. The present study is an evaluation of the effectiveness and safety of pazopanib treatment in patients with HHT treated on this pathway.

\section{Methods}

\section{Patients, data collection, and definitions}

This study was approved by the Institutional Review Board of the Cleveland Clinic. All patients with definite HHT (per the Curaçao criteria [2]) with severe HHT-associated bleeding and RBC transfusion dependence treated on the institutional Cleveland Clinic oral pazopanib treatment pathway (initiated in 2016) for one year or longer as of November 1 ,
2020 were identified and included, with data for analysis collected retrospectively. This predefined institutional pathway outlined the eligibility, dosing, visit schedule, laboratory and adverse event monitoring, and endpoints of treatment and their assessment prior to pazopanib treatment but was not a formal clinical trial protocol. RBC transfusion dependence and independence were defined using the Gale criteria, with dependence defined as $\geq 2$ units/month for $\geq 3$ months and independence defined as freedom from all RBC transfusion for $\geq 3$ months [24]. HHT-related GI bleeding was defined as known endoscopically visualized bleeding from gut mucosal telangiectasias. In addition to baseline demographics and HHT characteristics, data were collected for safety and effectiveness measures as detailed below.

\section{Pazopanib treatment pathway}

Patients were eligible for the pazopanib treatment pathway if they (1) had failed prior local hemostatic procedures and surgeries, systemic antifibrinolytics, and/or systemic bevacizumab; (2) were RBC transfusion-dependent per Gale criteria; (3) had no contraindications to pazopanib after an initial history, physical exam, and laboratory evaluation (Fig. 1); and (4) had not received an alternative systemic anti-angiogenic agent (such as bevacizumab, thalidomide, lenalidomide, or pomalidomide), bone marrow suppressive agents, or an investigational drug within 4 weeks of treatment initiation. Enrolled patients were started at a dose of $50 \mathrm{mg}$ daily. Dose escalation by $50-100 \mathrm{mg}$ increments (to a maximal dose of $400 \mathrm{mg}$ daily, Fig. 1) was performed at 30-day intervals (through either an in-person visit or telephone contact) to achieve a patient's optimal dose, defined as the lowest dose resulting in RBC transfusion independence and achieving an epistaxis severity score (ESS) in or near the mild range $(<4$ out of 10$)$ over the preceding month. Similarly, dose reduction to a minimum dose of $25 \mathrm{mg}$ daily was considered in patients achieving these milestones at the starting dose of $50 \mathrm{mg}$ daily or those developing a serious treatment-emergent adverse event (TEAE) determined to be related to pazopanib while receiving the starting dose of 50 mg daily. Patients were instructed to take pazopanib in the morning without food. Due to the negative impact from concomitant administration of gastric acid-suppressing agents with pazopanib in malignancy outcomes [25], all proton pump inhibitors were withdrawn prior to pazopanib administration. If patients' medical therapy for symptomatic gastroesophageal reflux disease was indicated, $\mathrm{H} 2$ antagonists were prescribed to be taken once daily in the evening along with dietary discretions.

Patients underwent follow-up clinical visits with vital signs, laboratory evaluations [complete blood count with white-cell differential, serum ferritin, iron and transferrin saturation, complete metabolic panel including liver function 


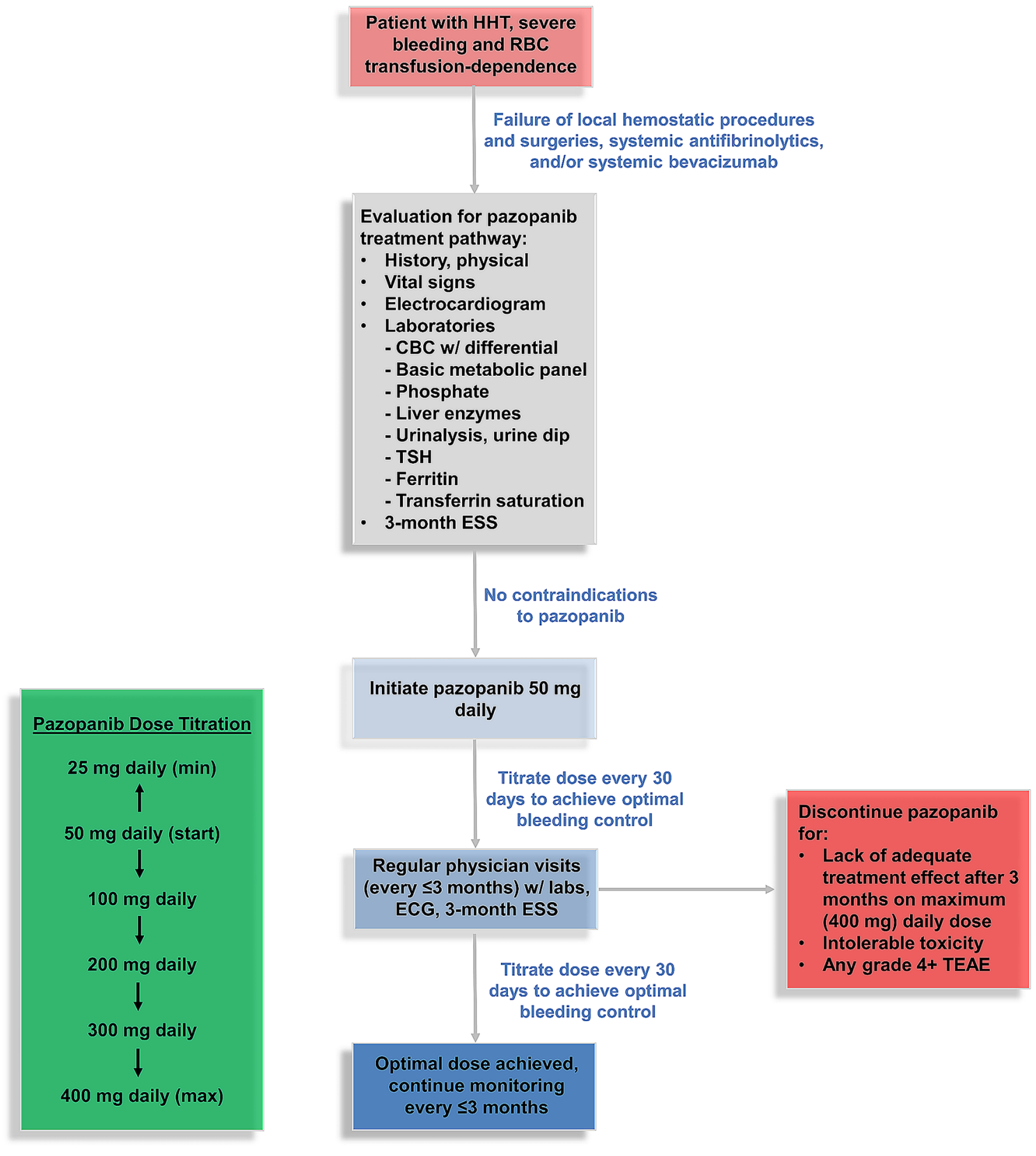

Fig. 1 Predefined pazopanib treatment pathway. Patients had required in-person clinic visits at least every 3 months and either telephone or in-person visits monthly (for drug titration and TEAE assessment).

testing (transaminases, bilirubin, and alkaline phosphatase), serum phosphorous, urinalysis, and thyroid-stimulating hormone levels], electrocardiogram, and epistaxis severity score (ESS) calculations every 3 months per the pathway, with additional laboratory testing and visits as indicated.

\section{Effectiveness measures of pazopanib}

All endpoints were evaluated over the first 12 months of treatment. The primary endpoint of the study was
$R B C$ red blood cell, $C B C$ complete blood count, $T S H$ thyroid-stimulating hormone, $E S S$ epistaxis severity score, $E C G$ electrocardiogram, $T E A E$ treatment-emergent adverse event

achievement of RBC transfusion independence per the Gale criteria during the first year of pazopanib treatment. Secondary endpoints were assessed as follows.

\section{Hemoglobin (Hgb)}

Baseline hemoglobin at pazopanib initiation was collected and compared with hemoglobin at each time point $(3,6$, 9 , and 12 months) on-treatment. Baseline and on-treatment hemoglobin values were drawn at clinical nadir (just prior to 
a scheduled transfusion) to minimize any impact of transfusion on the value. If more than one value was measured in a given 3-month period, the average value for that period was documented for analysis.

\section{Epistaxis severity score (ESS)}

The ESS is a well-validated continuous 10-point clinical score to longitudinally assess the severity of nosebleeds in patients with HHT [26]. A score of 0.00-1.00 signifies minimal or no epistaxis, 1.01-4.00 signifies mild epistaxis, 4.01-7.00 signifies moderate epistaxis, and 7.01-10.00 signifies severe epistaxis occurring over a specified time period. The minimal clinically important difference of the ESS is a change of 0.71 [27]. Three-month ESS was collected by the treating physician at treatment initiation and visits at 3 , 6, 9, and 12 months on-treatment. Baseline 3-month ESS at pazopanib initiation was collected and compared with 3-month ESS at each time point (3, 6, 9, and 12 months) on-treatment.

\section{$\mathrm{RBC}$ transfusion and iron infusion}

Number of RBC units transfused in the 3 months of pretreatment were compared with the number of units transfused at each 3-month time interval on-treatment (1-3, 4-6, 7-9, and 10-12 months). The same analysis was performed for the total milligrams of elemental iron administered by IV infusion.

\section{Iron studies}

Baseline serum ferritin and transferrin saturation at pazopanib initiation was collected and compared with ontreatment values (average of values collected at 6 and 12 months). Baseline and on-treatment iron studies were drawn at nadir (prior to iron infusion or RBC transfusion, if either was given) to minimize any impact of recent iron infusion or RBC transfusion on the values.

\section{Requirements for local hemostatic procedures}

The number and type of invasive nasal and endoscopic hemostatic procedures performed to treat epistaxis and/or GI bleeding in the 12 months prior to and during the first year of pazopanib treatment were captured and compared.

\section{Safety analysis}

Safety was evaluated for the entire duration of treatment for each patient and TEAEs were defined according to Common Terminology Criteria for Adverse Events v. 5.0 (National Cancer Institute). Specifically, patients were evaluated for
TEAEs by the treating physician every 30 days, during both in-person clinic visits and telephone contacts in between study visits for drug titration.

\section{Statistical analysis}

Data for each effectiveness measure were evaluated using paired means tests to compare baseline/pretreatment values with on-treatment values. The D'Agostino and Pearson normality test was applied to the data for each effectiveness measure to determine the appropriate hypothesis test used for that measure. This resulted in the use of the paired $t$ test (comparing 2 time points) or repeated measures one-way analysis of variance (ANOVA) with a Geisser-Greenhouse correction (comparing 3 or more time points) for hemoglobin and ESS because all data sets were parametric and Wilcoxon signed-rank test (comparing 2 time points) or Friedman test (comparing 3 or more time points) for other outcome measures because one or more data sets were nonparametric. When a repeated measures ANOVA showed a significant difference in means between time points, pairwise comparisons with a Bonferroni correction were performed to compare baseline/pretreatment mean with on-treatment means for each time point. Similarly, Dunn's multiple comparisons test was used to compare baseline/pretreatment mean with on-treatment means for each time point when a Friedman test showed a significant difference in means between time points.

\section{Results}

\section{Patient characteristics}

Sixteen transfusion-dependent adults with HHT were treated with oral pazopanib for epistaxis and/or GI bleeding; thirteen had been treated for at least 12 months and were included in the analysis (Fig. 2). All 3 patients excluded from the analysis were excluded because they had been treated for less than 12 months; pazopanib treatment was ongoing in 2 patients and discontinued in 1 patient due to worsening of baseline leukopenia (additional details given in the Supplementary Appendix). In the 13 included patients, the median age was 66 (range, 53-78) years and 46\% were female. All patients had complete and continuous follow-up during their treatment duration and data collection for endpoints and safety was complete. Table 1 lists baseline patient characteristics. Patients were treated for a median (range) of 22 (13-46) months, for a total of 25.4 patient-years of pazopanib treatment in the cohort. The predominant source of chronic bleeding was epistaxis in $6(46 \%)$, GI bleeding in $3(23 \%)$, and both in $4(31 \%)$ patients. No patient had a complicating additional etiology of anemia or had received 


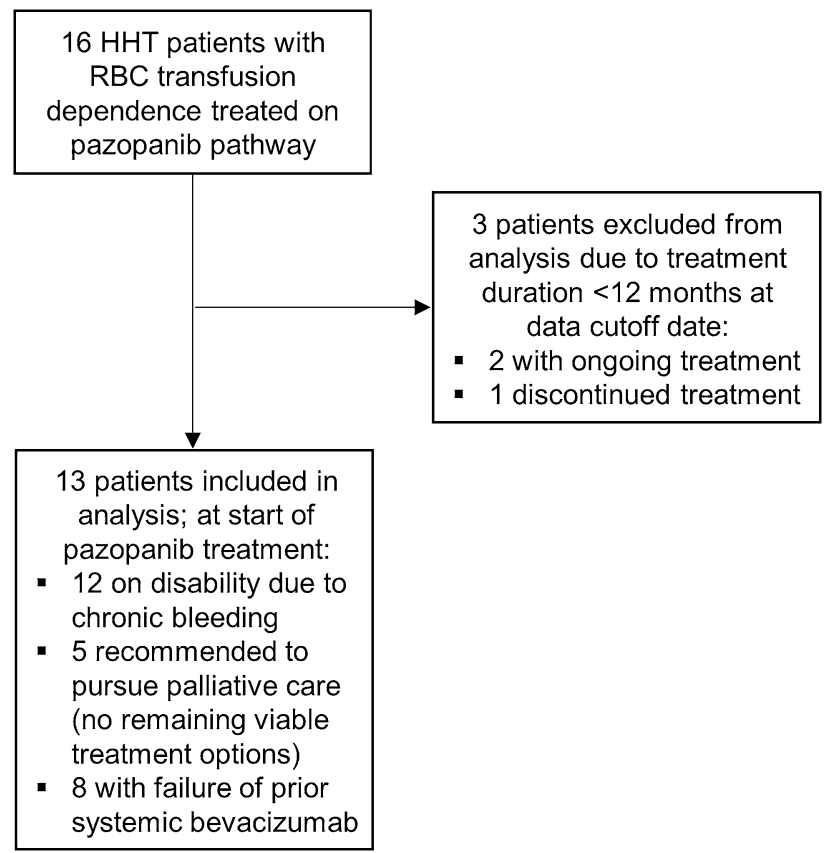

Fig. 2 Flow diagram describing patient inclusion and exclusion in study analysis

other hemostatic agents (such as antifibrinolytics or hormonal agents) or erythropoiesis-stimulating agents in the 6 months prior to pathway enrollment or at any point during pazopanib treatment.

All patients began receiving intermittent RBC transfusions at a median of 37 (13-73) months prior to initiating pazopanib, with progression to transfusion dependence a median of 11 (5-18) months prior. Twelve patients (92\%) were on medical disability due to their chronic bleeding and
$5(38 \%)$ were recommended to pursue palliative care as all available therapeutic options had been explored. Eight of 13 patients were treated with pazopanib following failure of prior systemic bevacizumab (3 patients with primary non-response after 3 months of prior bevacizumab treatment and 5 patients with loss of bevacizumab response after 13-35 months of prior bevacizumab treatment). Primary non-responders had no significant reduction in bleeding following receipt of a bevacizumab induction regimen of $5 \mathrm{mg} /$ $\mathrm{kg}$ every 2 weeks for 6 infusions. Patients with loss of bevacizumab response became RBC transfusion-dependent (per the Gale criteria) despite ongoing treatment. All of these patients had previously undergone bevacizumab induction as previously described and were receiving continuous bevacizumab maintenance with $5 \mathrm{mg} / \mathrm{kg}$ bevacizumab administered every $2-4$ weeks. All patients previously receiving bevacizumab had $a \geq 4$-week washout period before initiating pazopanib. Seven patients had historically been treated with and failed tranexamic acid (1300 mg BID or TID); no patient had previously received hormone-based bleeding treatment or erythropoiesis-stimulating agents. Seven patients had signs or symptoms of high-output cardiac failure (described in the Supplementary Appendix).

\section{Pazopanib dosing}

The median (range) daily optimal pazopanib dose was $100 \mathrm{mg}(25-300 \mathrm{mg})$ and no patient required dose escalation to the pre-specified maximum dose of $400 \mathrm{mg}$ daily. The median (range) optimal pazopanib dose in patients whose predominant bleeding source was epistaxis was $200 \mathrm{mg}$ (100-300 mg) daily, higher than the $100 \mathrm{mg}(25-100 \mathrm{mg})$ patients with a predominant bleeding source of GI bleeding.

Table 1 Baseline characteristics of HHT patients treated with pazopanib for severe bleeding and transfusion-dependent iron deficiency anemia

\begin{tabular}{|c|c|c|c|c|}
\hline No. & Age & Sex & Genetic mutation & Local hemostatic procedures in 12 months of pretreatment \\
\hline 1. & 71 & $\mathrm{~F}$ & ENG & Partial nasal closure (Young's procedure), nasal electrocautery $(\times 2)$ \\
\hline 2. & 67 & $\mathrm{~F}$ & ACVRL1 & Laser photocoagulation $(\times 2)$ \\
\hline 3. & 53 & M & ENG & $\begin{array}{l}\text { Laser photocoagulation }(\times 2) \text {, nasal arterial embolization, nasal electrocautery, sub- } \\
\text { mucosal nasal bevacizumab }\end{array}$ \\
\hline 4. & 67 & M & ACVRL1 & Submucosal nasal bevacizumab $(\times 2)$, nasal electrocautery $(\times 2)$ \\
\hline 5. & 62 & M & ACVRL1 & Argon plasma coagulation $(\times 4)$ \\
\hline 6. & 78 & $\mathrm{~F}$ & ENG & Submucosal nasal bevacizumab, nasal electrocautery, argon plasma coagulation $(\times 3)$ \\
\hline 7. & 71 & M & ENG & Argon plasma coagulation $(\times 3)$, endoscopic clip ligation \\
\hline 8. & 60 & M & ENG & Laser photocoagulation, nasal electrocautery, argon plasma coagulation $(\times 3)$ \\
\hline 9. & 65 & $\mathrm{~F}$ & ACVRL1 & Laser photocoagulation $\times 2$, nasal electrocautery \\
\hline 10. & 67 & M & Unknown & Argon plasma coagulation $(\times 4)$ \\
\hline 11. & 54 & $\mathrm{~F}$ & Unknown & Argon plasma coagulation \\
\hline 12. & 67 & $\mathrm{~F}$ & ACVRL1 & Nasal arterial embolization, nasal electrocautery, argon plasma coagulation $(\times 2)$ \\
\hline 13. & 70 & M & ACVRL1 & $\begin{array}{l}\text { Laser photocoagulation, nasal arterial embolization, nasal electrocautery }(\times 2) \text {, } \\
\text { submucosal bevacizumab }\end{array}$ \\
\hline
\end{tabular}


Drug was obtained via the following mechanisms: 4 patients obtained insurance approval; 4 patients purchased drug out of pocket; 5 patients received free drug from the manufacturer.

\section{Effectiveness measures of pazopanib}

\section{Primary endpoint}

All 13 patients achieved RBC transfusion independence, with 10 patients (77\%) achieving transfusion independence at 3 months of treatment and the remaining 3 patients $(23 \%)$ achieving transfusion independence at 6 months of treatment. Nine patients $(69 \%)$ were entirely RBC transfusionfree during the 12 months following pazopanib initiation.

\section{Hemoglobin}

All patients were anemic at baseline, with a mean $\mathrm{Hgb}$ of 7.8 (95\% CI, 7.4-8.4) g/dL. Mean hemoglobin was significantly different over the course of treatment $[F(2.129$, $25.55)=83.65, P<0.0001)]$, with increases of $2.0(95 \% \mathrm{CI}$, 1.3-2.7), 3.9 (95\% CI, 2.8-5.1), 4.6 (95\% CI, 3.4-5.7), and 4.8 (95\% CI, 3.6-5.9) g/dL at 3, 6, 9, and 12 months, respectively, relative with baseline (adjusted $P<0.0001$ for all pairwise comparisons). Freedom from anemia $(\mathrm{Hgb} \geq 11 \mathrm{~g} /$ dL) was observed in $15 \%$ of patients at 3 months, $85 \%$ at 6 months, $85 \%$ at 9 months, and $92 \%$ at 12 months (Fig. 3A).

\section{Epistaxis severity score}

The mean ESS at baseline was 7.20 (95\% CI, 5.58-8.82) points, with 9 patients $(69 \%)$ in the severe range. Mean ESS was significantly different over the course of treatment $[F(1.295,15.54)=62.43, P<0.0001)]$, with decreases of 2.57 (95\% CI, 1.77-3.36), 3.80 (95\% CI, 2.62-4.98), 4.76 (95\% CI, 3.10-6.42), and 4.77 (95\% CI, 3.11-6.44) points at $3,6,9$, and 12 months, respectively, relative with baseline (adjusted $P<0.0001$ for all pairwise comparisons). A clinically meaningful reduction in epistaxis (ESS decrease of $\geq 0.71$ post treatment) was observed in all patients at 3 months and maintained at 6, 9, and 12 months for all patients (Fig. 3B).

\section{RBC transfusion}

All patients were transfusion-dependent at baseline (range, 8-28 RBC units transfused in the 3 months of pretreatment). There was a significant difference in RBC units transfused over the course of treatment $\left[\chi^{2}(4)=43.80\right.$, $P<0.0001)$ ], with a decrease in transfusions from a median (IQR) of 16.0 (9.5-22.0) RBC units in the 3 months of pretreatment to a median (IQR) of $0.0(0.0-1.0)$ units at
1-3 months of on-treatment and $0.0(0.0-0.0)$ at 4-6, 7-9, and 10-12 months of on-treatment (adjusted $P<0.001$ for all pairwise comparisons), Fig. 3C. RBC transfusion events for each patient prior to and following pazopanib treatment are illustrated in Fig. 4.

\section{Iron infusion}

There was a significant difference in milligrams of elemental iron infused over the course of treatment $\left[\chi^{2}(4)=31.66\right.$, $P<0.0001)]$, with a decrease in elemental iron infused from a median (IQR) of 4500 (2145-7260) milligrams in the 3 months of pretreatment to a median (IQR) of $0(0-1500)$ milligrams at $1-3$ months, $0(0-1260)$ at 4-6 months, 0 $(0-1260)$ at $7-9$ months, and $0(0-510)$ at 10-12 months of on-treatment (adjusted $P<0.005$ for all pairwise comparisons), Fig. 3D. Iron infusion events for each patient prior to and following pazopanib treatment are illustrated in Fig. 4. Of note, all patients were also receiving continuous oral iron supplementation (65 mg elemental iron 1-3 times daily) throughout the 3-month pretreatment period and during the 12 months on pazopanib treatment.

\section{Serum ferritin}

Median (IQR) ferritin improved from $31(16-71) \mathrm{ng} / \mathrm{mL}$ at baseline to $122(79-154) \mathrm{ng} / \mathrm{mL}$ on-treatment $(P=0.0007)$, Fig. 3E. Nine patients (69\%) had a baseline ferritin $<40 \mathrm{ng} /$ $\mathrm{dL}$; none had a ferritin $<40 \mathrm{ng} / \mathrm{dL}$ at month 6 or 12 on-treatment.

\section{Serum transferrin saturation}

Median (IQR) transferrin saturation improved from $9 \%(5-11 \%)$ at baseline to $23 \%(16-31 \%)$ on-treatment $(P=0.0002)$, Fig. 3F. No patient had a transferrin saturation $\geq 20 \%$ at baseline, compared with 8 patients $(62 \%)$ who did at 6 months and 7 patients (54\%) who did at 12 months.

\section{Requirement for local hemostatic procedures}

The number of local hemostatic procedures decreased from a median (range) of 4 (1-5) in the 12 months of pretreatment to $0(0-2)$ during the 12 months of on-treatment $(P=0.0002)$, with 9 patients $(69 \%)$ not requiring any procedures after initiating treatment. Of the 4 patients undergoing procedures, 3 underwent nasal sclerotherapy once and 1 underwent nasal sclerotherapy twice (Supplemental Table 1). 


\section{A}

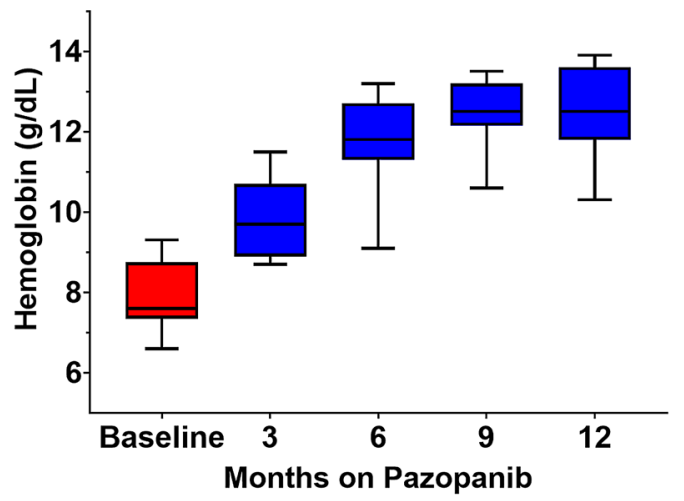

C

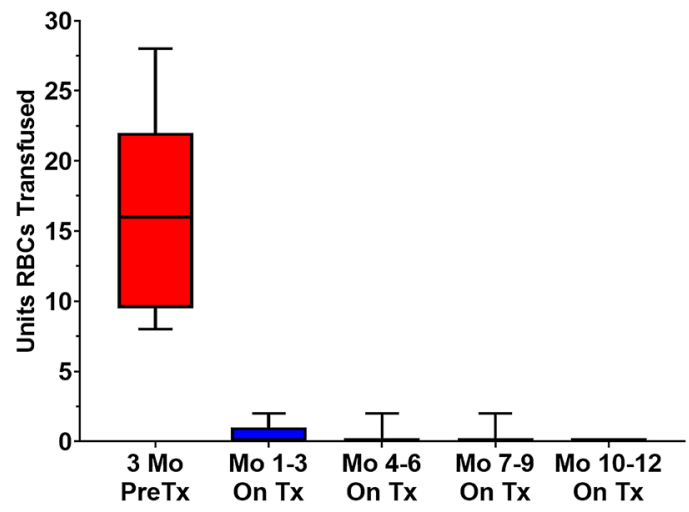

$\mathbf{E}$

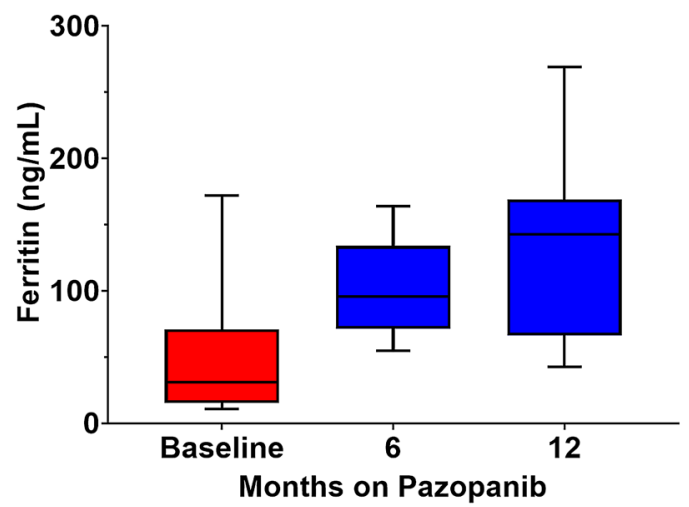

Fig. 3 Box-and-whisker plots (box represents median and interquartile range and tails represent minimum and maximum) showing effect of pazopanib on hematologic parameters and epistaxis sever-

\section{Safety analysis}

TEAEs possibly or likely related to pazopanib treatment are summarized in Table 2 (TEAEs in patients not included

\section{B}

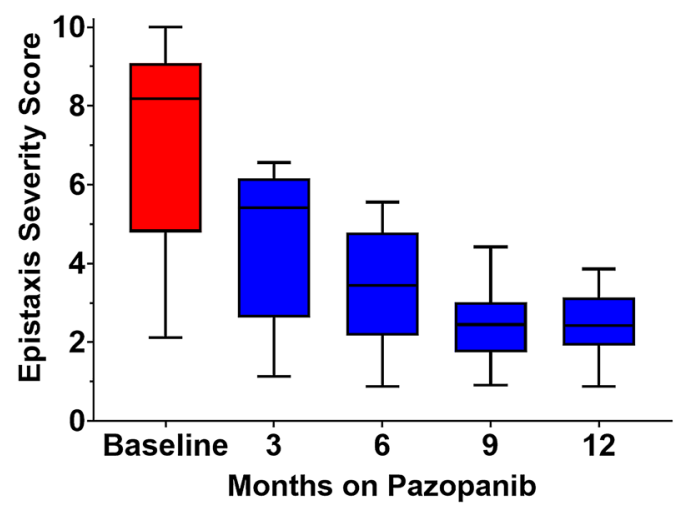

D

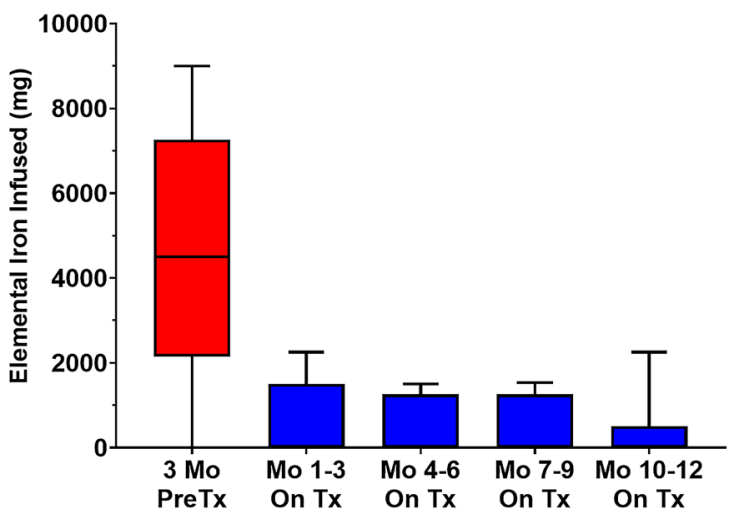

$\mathbf{F}$

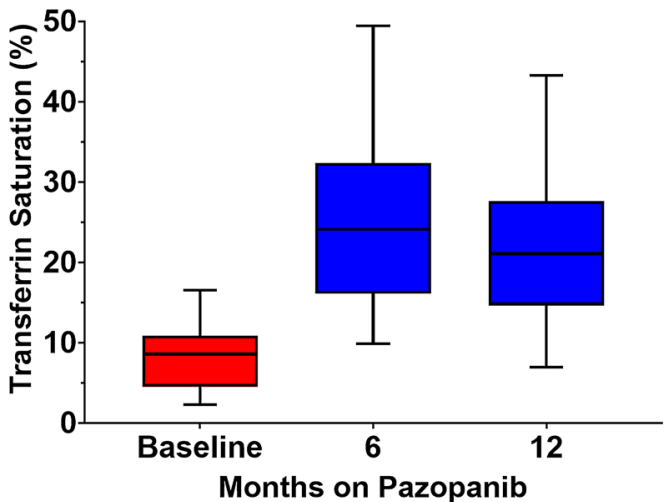

ity. A Hemoglobin, B Epistaxis severity score, C RBC transfusion, D Iron infusion, E Serum ferritin, $\mathbf{F}$ Transferrin saturation. Mo months, PreTx pretreatment, On Tx on-treatment

in the primary analysis are detailed in the Supplementary Appendix). The most common TEAEs were hypertension (31\%), lymphocytopenia (23\%), and fatigue (23\%). No 


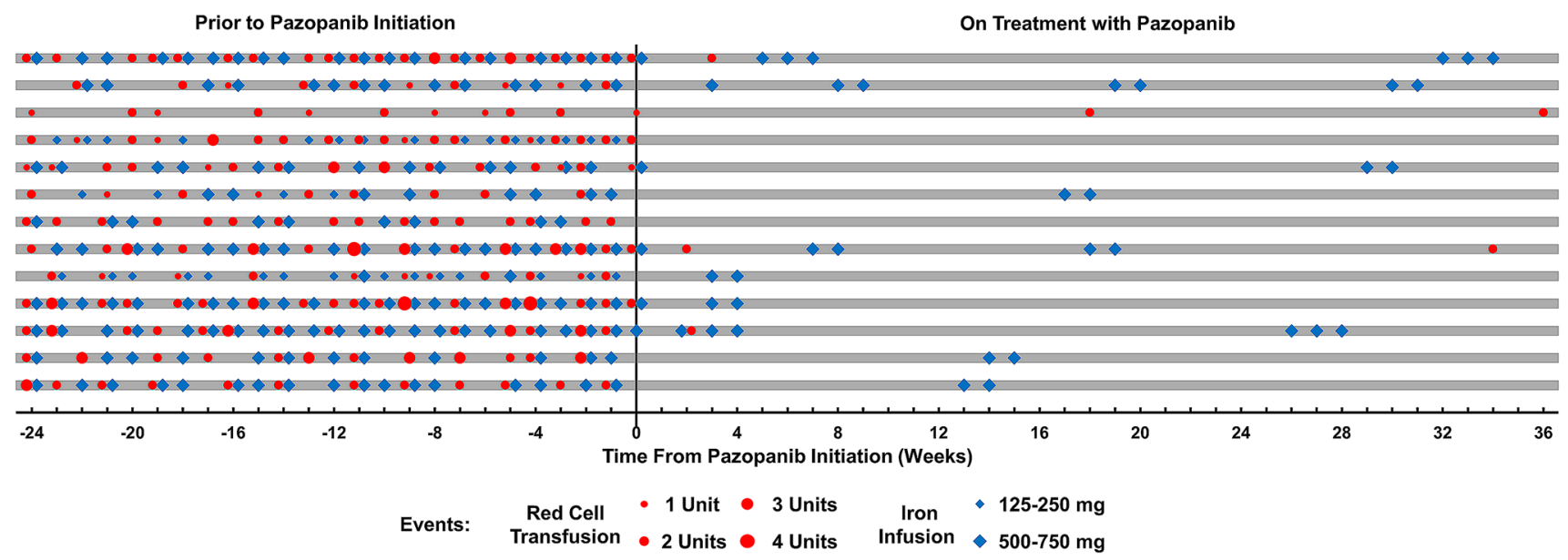

Fig. 4 Swimmer plot detailing timeline of red cell transfusion and iron infusion events for each patient during the 24 months prior to pazopanib initiation and 36 weeks following its initiation

Table 2 Treatment-emergent adverse events (TEAEs) identified as likely or possibly due to pazopanib in the 13-patient cohort

\begin{tabular}{|c|c|c|}
\hline TEAE & $\begin{array}{l}\text { CTCAE v } 5.0 \\
\text { grade* }\end{array}$ & No. of patients $(\%)$ \\
\hline Hypertension & 2 & $4(31 \%)$ \\
\hline \multirow[t]{2}{*}{ Lymphocytopenia } & 1 & $2(15 \%)$ \\
\hline & 2 & $1(8 \%)$ \\
\hline Fatigue & 1 & $3(23 \%)$ \\
\hline Headache & 1 & $2(15 \%)$ \\
\hline Dyspepsia & 1 & $2(15 \%)$ \\
\hline Hypothyroidism ${ }^{\dagger}$ & 2 & $2(15 \%)$ \\
\hline Dysgeusia & 1 & $1(8 \%)$ \\
\hline Hypophosphatemia & 2 & $1(8 \%)$ \\
\hline $\begin{array}{l}\text { Benign migratory glossitis } \\
\text { (geographic tongue) }\end{array}$ & 1 & $1(8 \%)$ \\
\hline
\end{tabular}

TEAEs observed in 3 patients excluded from the primary analysis are detailed in the Supplementary Appendix

*Where multiple patients had a TEAE and one grade is given, this grade applied to all patients with that TEAE

${ }^{\dagger}$ Both patients developing hypothyroidism as a TEAE had pre-existing hypothyroidism, with worsening requiring uptitration of thyroid replacement after initiating pazopanib

patient developed LFT abnormalities, venous or arterial thromboembolism, proteinuria or QTc prolongation during treatment. Overall, 12 patients (92\%) experienced at least 1 TEAE, however, none required treatment discontinuation or dose reduction. There were no fatal TEAEs, although one patient, a 78-year-old female, died of viral pneumonia attributed to coronavirus disease 2019 (COVID-19) after treatment with pazopanib for 15 months. This patient had developed grade 2 lymphocytopenia on pazopanib treatment prior to contracting COVID-19 (absolute lymphocyte count $645 / \mathrm{mm}^{3}$ ), but had no clinical or radiographic evidence of drug-induced pneumonitis prior to or during this acute illness.

\section{Discussion}

We present the findings of a study evaluating treatment outcomes of a single-center institutional pazopanib treatment pathway for severe bleeding and transfusion-dependent anemia in patients with HHT. All 13 patients treated met the primary endpoint of achieving RBC transfusion independence per the Gale criteria within 12 months of treatment initiation and significant improvements in all secondary endpoints were observed, including striking improvements in hemoglobin, ESS, RBC transfusions, iron infusions, iron stores, and invasive hemostatic procedures (Figs. 3, 4). Total freedom from RBC transfusions after pazopanib initiation was achieved in over two-thirds of patients. Overall, substantial hemostasis was achieved by 3 months of treatment, improved further at the 6-month mark and was sustained through the 9- and 12-month time points. Whereas all patients required one or more invasive hemostatic procedures in the year prior to treatment, $69 \%$ required no such procedures after starting pazopanib. Pazopanib was safe overall, with an acceptable incidence of grade 1-2 TEAEs.

Prior data describing the use of pazopanib in HHT are limited to a single-patient case report [22] and a phase I pilot study of 7 patients treated for 12 weeks, of which only 1 was transfusion-dependent (as in the present study, this resolved with pazopanib treatment) [23]. The average patient in this pilot study had considerably less severe bleeding than the current study, with a mean baseline ESS at 4.47 (just above 
the mild range) and a mean baseline hemoglobin of $10.1 \mathrm{gm} /$ dL. Despite treatment with a low dose of $50 \mathrm{mg}$ pazopanib for 12 weeks, most patients experienced improvements in both ESS and hemoglobin levels. Including a total of 1.6 patient-years of pazopanib treatment, all adverse events were mild to moderate except for an occurrence of grade 3 alanine aminotransferase elevation in one patient, resulting in discontinuation of pazopanib. The present study found similar overall safety over a much longer period of 25.4 patient-years of pazopanib treatment. Most TEAEs were modest. Pazopanib has a well-known impact on blood pressure, and this was observed in 4 patients $(31 \%)$ where it was effectively treated with antihypertensive medications. No liver function test changes were recorded, and mild thyroid function test abnormalities necessitating thyroid replacement dose adjustments were found in two (15\%) patients with established hypothyroidism. One patient, who was not included in the primary analysis because of a treatment duration of $<12$ months, had pazopanib discontinued for worsening of baseline leukopenia (grade 1 at baseline to grade 3 after 9 months of pazopanib) with recovery to baseline after pazopanib discontinuation. Although patients were treated for up to 3.8 years in this study without any apparent TEAEs associated with extended-duration treatment, the impact of extended pazopanib exposure in HHT (either positive or negative) is not known.

Other anti-angiogenic agents with data published for the treatment of HHT-associated bleeding include bevacizumab [15-18], thalidomide [28], and pomalidomide [29], with the largest amount of data supporting intravenous bevacizumab. Recent studies have also highlighted the anti-angiogenic potential of the antifungal itraconazole [30] and the local hemostatic capacity of intranasal fluorouracil (Efudix) [31] in HHT. The data supporting intravenous bevacizumab were sufficient for its recommendation to manage moderate-tosevere epistaxis and/or GI bleeding in the recently published updated international HHT guidelines [32]. By any measure, the outcomes of patients treated with pazopanib in the present study are comparable with the outcomes of bevacizumab use in the InHIBIT-Bleed study, the largest study of bevacizumab for chronic bleeding in HHT [15]. Pazopanib has several intrinsic advantages to bevacizumab, most notably its oral mode of administration, thereby obviating the inconvenience, cost, and infusion center infrastructure required for repeated and indefinite intravenous infusions. Another significant advantage of pazopanib is that liberation from transfusion dependence was achieved at doses much lower than typically used for oncologic indications, in contrast to bevacizumab which is often prescribed at doses at or near those used to treat malignancies. This is especially relevant with pazopanib treatment, as pazopanib toxicity has clearly been demonstrated to be dose-dependent [33]. Additionally, most patients in this study had previously failed systemic bevacizumab and yet responded favorably to pazopanib. Even in light of the data we present from this study, it is important to recognize that the published data for pazopanib treatment of HHT remain limited. Therefore, we advise caution with use of this agent to treat HHT outside of a clinical trial setting, which if done should be overseen by an HHT expert.

Although the number of patients treated for predominant GI bleeding was small in this study, the doses required in these patients were generally lower than the doses required for patients with predominant epistaxis. This finding is supported by the murine model that showed that orally delivered anti-angiogenic tyrosine kinase inhibitors are selectively more effective for GI bleeding than they are for mucocutaneous nasal AVMs causing epistaxis [21]. This finding is further supported by the observation that the 4 patients needing invasive hemostatic procedures on pazopanib therapy were for epistaxis control.

Our study has many strengths but also a number of limitations. Although patients were enrolled onto a predefined pathway that prospectively outlined management and facilitated completeness of collected data, they were not enrolled onto a formal treatment protocol. While data for analysis were collected retrospectively, relevant data were generated over the course of treatment and data collection was complete owing to treatment on this predefined pathway. While the lack of randomization allows for the presence of confounding factors that could have resulted in clinical improvements independent of pazopanib use, the substantial improvements across all outcome measures that occurred coinciding with initiation of pazopanib treatment greatly increases our confidence that pazopanib was the likely cause of these improvements. To minimize patient heterogeneity intrinsic to HHT, we employed a pre/post-treatment analytical design with paired and repeated measures analyses such that each patient served as his/her own internal control in effectiveness analyses. Adverse event reporting was limited by the lack of enrollment onto a formal prospective protocol, and so it is possible some non-serious adverse events may have been missed. But given complete and continuous follow-up of patients, we expect it is unlikely that any serious adverse events would not have been captured.

In conclusion, we observed that oral pazopanib was effective in the management of transfusion-dependent HHT patients with severe bleeding. All treated patients were liberated from transfusion dependence and had near-complete normalization of hematologic parameters. Pazopanib was well tolerated over 25.4 patient-years of treatment. Larger, randomized studies are needed to confirm these findings and better define the role of low-dose pazopanib in the treatment of bleeding in HHT. 
Supplementary Information The online version contains supplementary material available at https://doi.org/10.1007/s10456-021-09807-4.

Acknowledgements H. Al-Samkari is the recipient of the Harvard KL2/Catalyst Medical Research Investigator Training Award and the American Society of Hematology Scholar Award.

Author contributions JP contributed to concept and design, data collection, writing the first draft of the manuscript, critical revision of the manuscript, and final approval; HA-S contributed to concept and design, writing the first draft of the manuscript, data analysis, creation of the tables and figures, critical revision of the manuscript, and final approval; JG, KM, and DS contributed to concept and design, critical revision of the manuscript, and final approval; all other authors contributed to critical revision of the manuscript and final approval.

Data availability The data sets generated during and/or analyzed during the current study are available from the corresponding author on reasonable request.

\section{Declarations}

Conflict of interest Al-Samkari: consultancy (Agios, Dova, Argenx, Rigel, Sobi, Novartis), Research Funding to Institution (Agios, Dova, Amgen). All other authors have nothing to disclose.

Ethical approval This study was approved by the Institutional Review Board of the Cleveland Clinic.

\section{References}

1. Kritharis A, Al-Samkari H, Kuter DJ (2018) Hereditary hemorrhagic telangiectasia: diagnosis and management from the hematologist's perspective. Haematologica. https://doi.org/10.3324/ haematol.2018.193003

2. Shovlin CL, Guttmacher AE, Buscarini E, Faughnan ME, Hyland RH, Westermann CJ, Kjeldsen AD, Plauchu H (2000) Diagnostic criteria for hereditary hemorrhagic telangiectasia (Rendu-OslerWeber syndrome). Am J Med Genet 91(1):66-67

3. Parambil JG (2016) Hereditary hemorrhagic telangiectasia. Clin Chest Med 37(3):513-521. https://doi.org/10.1016/j.ccm.2016.04. 013

4. Shovlin CL (2010) Hereditary haemorrhagic telangiectasia: pathophysiology, diagnosis and treatment. Blood Rev 24(6):203-219. https://doi.org/10.1016/j.blre.2010.07.001

5. Letteboer TG, Mager HJ, Snijder RJ, Lindhout D, Ploos van Amstel HK, Zanen P, Westermann KJ (2008) Genotype-phenotype relationship for localization and age distribution of telangiectases in hereditary hemorrhagic telangiectasia. Am J Med Genet A 146A(21):2733-2739. https://doi.org/10.1002/ajmg.a.32243

6. Kasthuri RS, Montifar M, Nelson J, Kim H, Lawton MT, Faughnan ME, Brain Vascular Malformation Consortium HHTIG (2017) Prevalence and predictors of anemia in hereditary hemorrhagic telangiectasia. Am J Hematol. https://doi.org/10.1002/ajh. 24832

7. Donaldson JW, McKeever TM, Hall IP, Hubbard RB, Fogarty AW (2015) Complications and mortality in hereditary hemorrhagic telangiectasia: a population-based study. Neurology 84(18):18861893. https://doi.org/10.1212/WNL.0000000000001538

8. Geisthoff UW, Heckmann K, D'Amelio R, Grunewald S, Knobber D, Falkai P, Konig J (2007) Health-related quality of life in hereditary hemorrhagic telangiectasia. Otolaryngol Head Neck Surg 136(5):726-733. https://doi.org/10.1016/j.otohns.2006.12. 019

9. Merlo CA, Yin LX, Hoag JB, Mitchell SE, Reh DD (2014) The effects of epistaxis on health-related quality of life in patients with hereditary hemorrhagic telangiectasia. Int Forum Allergy Rhinol 4(11):921-925. https://doi.org/10.1002/alr.21374

10. Cirulli A, Liso A, D’Ovidio F, Mestice A, Pasculli G, Gallitelli M, Rizzi R, Specchia G, Sabba C (2003) Vascular endothelial growth factor serum levels are elevated in patients with hereditary hemorrhagic telangiectasia. Acta Haematol 110(1):29-32. https:// doi.org/10.1159/000072411

11. Sadick H, Riedel F, Naim R, Goessler U, Hormann K, Hafner M, Lux A (2005) Patients with hereditary hemorrhagic telangiectasia have increased plasma levels of vascular endothelial growth factor and transforming growth factor-beta1 as well as high ALK1 tissue expression. Haematologica 90(6):818-828

12. Al-Samkari H (2020) Hereditary hemorrhagic telangiectasia: systemic therapies, guidelines, and an evolving standard of care. Blood. https://doi.org/10.1182/blood.2020008739

13. Al-Samkari H, Albitar HA, Olitsky SE, Clancy MS, Iyer VN (2019) Systemic bevacizumab for high-output cardiac failure in hereditary hemorrhagic telangiectasia: an international survey of HHT centers. Orphanet J Rare Dis 14(1):256. https://doi.org/10. 1186/s13023-019-1239-6

14. Al-Samkari H, Albitar HA, Olitsky SE, Clancy MS, Iyer VN (2020) An international survey to evaluate systemic bevacizumab for chronic bleeding in hereditary haemorrhagic telangiectasia. Haemophilia. https://doi.org/10.1111/hae.14034

15. Al-Samkari H, Kasthuri RS, Parambil JG, Albitar HA, Almodallal YA, Vazquez C, Serra MM, Dupuis-Girod S, Wilsen CB, McWilliams JP, Fountain EH, Gossage JR, Weiss CR, Latif MA, Issachar A, Mei-Zahav M, Meek ME, Conrad M, Rodriguez-Lopez J, Kuter DJ, Iyer VN (2020) An international, multicenter study of intravenous bevacizumab for bleeding in hereditary hemorrhagic telangiectasia: the InHIBIT-Bleed study. Haematologica. https:// doi.org/10.3324/haematol.2020.261859

16. Al-Samkari H, Kritharis A, Rodriguez-Lopez JM, Kuter DJ (2019) Systemic bevacizumab for the treatment of chronic bleeding in hereditary haemorrhagic telangiectasia. J Intern Med 285(2):223231. https://doi.org/10.1111/joim. 12832

17. Iyer VN, Apala DR, Pannu BS, Kotecha A, Brinjikji W, Leise MD, Kamath PS, Misra S, Begna KH, Cartin-Ceba R, DuBrock HM, Krowka MJ, O'Brien EK, Pruthi RK, Schroeder DR, Swanson KL (2018) Intravenous bevacizumab for refractory hereditary hemorrhagic telangiectasia-related epistaxis and gastrointestinal bleeding. Mayo Clin Proc 93(2):155-166. https://doi.org/10.1016/j. mayocp.2017.11.013

18. Dupuis-Girod S, Ginon I, Saurin JC, Marion D, Guillot E, Decullier E, Roux A, Carette MF, Gilbert-Dussardier B, Hatron PY, Lacombe P, Lorcerie B, Riviere S, Corre R, Giraud S, Bailly S, Paintaud G, Ternant D, Valette PJ, Plauchu H, Faure F (2012) Bevacizumab in patients with hereditary hemorrhagic telangiectasia and severe hepatic vascular malformations and high cardiac output. JAMA 307(9):948-955. https://doi.org/10.1001/jama. 2012.250

19. Buscarini E, Botella LM, Geisthoff U, Kjeldsen AD, Mager HJ, Pagella F, Suppressa P, Zarrabeitia R, Dupuis-Girod S, Shovlin CL, Vascern HHT (2019) Safety of thalidomide and bevacizumab in patients with hereditary hemorrhagic telangiectasia. Orphanet J Rare Dis 14(1):28. https://doi.org/10.1186/s13023-018-0982-4

20. Schutz FA, Choueiri TK, Sternberg CN (2011) Pazopanib: clinical development of a potent anti-angiogenic drug. Crit Rev Oncol Hematol 77(3):163-171. https://doi.org/10.1016/j.critrevonc. 2010.02 .012 
21. Kim YH, Kim MJ, Choe SW, Sprecher D, Lee YJ (2017) Selective effects of oral antiangiogenic tyrosine kinase inhibitors on an animal model of hereditary hemorrhagic telangiectasia. J Thromb Haemost 15(6):1095-1102. https://doi.org/10.1111/jth.13683

22. Parambil JG, Woodard TD, Koc ON (2018) Pazopanib effective for bevacizumab-unresponsive epistaxis in hereditary hemorrhagic telangiectasia. Laryngoscope 128(10):2234-2236. https:// doi.org/10.1002/lary.27129

23. Faughnan ME, Gossage JR, Chakinala MM, Oh SP, Kasthuri R, Hughes CCW, McWilliams JP, Parambil JG, Vozoris N, Donaldson J, Paul G, Berry P, Sprecher DL (2019) Pazopanib may reduce bleeding in hereditary hemorrhagic telangiectasia. Angiogenesis 22(1):145-155. https://doi.org/10.1007/s10456-018-9646-1

24. Gale RP, Barosi G, Barbui T, Cervantes F, Dohner K, Dupriez B, Gupta V, Harrison C, Hoffman R, Kiladjian JJ, Mesa R, Mc Mullin MF, Passamonti F, Ribrag V, Roboz G, Saglio G, Vannucchi A, Verstovsek S (2011) What are RBC-transfusion-dependence and -independence? Leuk Res 35(1):8-11. https://doi.org/10.1016/j. leukres.2010.07.015

25. Mir O, Touati N, Lia M, Litiere S, Le Cesne A, Sleijfer S, Blay JY, Leahy M, Young R, Mathijssen RHJ, Van Erp NP, Gelderblom H, Van der Graaf WT, Gronchi A (2019) Impact of concomitant administration of gastric acid-suppressive agents and pazopanib on outcomes in soft-tissue sarcoma patients treated within the EORTC 62043/62072 trials. Clin Cancer Res 25(5):1479-1485. https://doi.org/10.1158/1078-0432.CCR-18-2748

26. Hoag JB, Terry P, Mitchell S, Reh D, Merlo CA (2010) An epistaxis severity score for hereditary hemorrhagic telangiectasia. Laryngoscope 120(4):838-843. https://doi.org/10.1002/lary. 20818

27. Yin LX, Reh DD, Hoag JB, Mitchell SE, Mathai SC, Robinson GM, Merlo CA (2016) The minimal important difference of the epistaxis severity score in hereditary hemorrhagic telangiectasia. Laryngoscope 126(5):1029-1032. https://doi.org/10.1002/lary. 25669

28. Invernizzi R, Quaglia F, Klersy C, Pagella F, Ornati F, Chu F, Matti E, Spinozzi G, Plumitallo S, Grignani P, Olivieri C, Bastia R, Bellistri F, Danesino C, Benazzo M, Balduini CL (2015)
Efficacy and safety of thalidomide for the treatment of severe recurrent epistaxis in hereditary haemorrhagic telangiectasia: results of a non-randomised, single-centre, phase 2 study. The Lancet Haematology 2(11):e465-473. https://doi.org/10.1016/ S2352-3026(15)00195-7

29. Samour M, Saygin C, Abdallah R, Kundu S, McCrae KR (2016) Pomalidomide in hereditary hemorrhagic telangiectasia: interim results of a phase I study. Blood 128(22):210

30. Kroon S, Snijder RJ, Hosman AE, Vorselaars VMM, Disch FJM, Post MC, Mager JJ (2020) Oral itraconazole for epistaxis in hereditary hemorrhagic telangiectasia: a proof of concept study. Angiogenesis. https://doi.org/10.1007/s10456-020-09758-2

31. de Jel DVC, Disch FJM, Kroon S, Mager JJ, Verdam FJ (2020) Intranasal Efudix reduces epistaxis in hereditary hemorrhagic telangiectasia. Angiogenesis 23(3):271-274. https://doi.org/10. 1007/s10456-020-09712-2

32. Faughnan ME, Mager JJ, Hetts SW, Palda VA, Lang-Robertson K, Buscarini E, Deslandres E, Kasthuri RS, Lausman A, Poetker D, Ratjen F, Chesnutt MS, Clancy M, Whitehead KJ, Al-Samkari H, Chakinala M, Conrad M, Cortes D, Crocione C, Darling J, de Gussem E, Derksen C, Dupuis-Girod S, Foy P, Geisthoff U, Gossage JR, Hammill A, Heimdal K, Henderson K, Iyer VN, Kjeldsen AD, Komiyama M, Korenblatt K, McDonald J, McMahon J, McWilliams J, Meek ME, Mei-Zahav M, Olitsky S, Palmer S, Pantalone R, Piccirillo JF, Plahn B, Porteous MEM, Post MC, Radovanovic I, Rochon PJ, Rodriguez-Lopez J, Sabba C, Serra M, Shovlin C, Sprecher D, White AJ, Winship I, Zarrabeitia R (2020) Second international guidelines for the diagnosis and management of hereditary hemorrhagic telangiectasia. Ann Intern Med. https:// doi.org/10.7326/M20-1443

33. FDA US Votrient (pazopanib) prescribing information. Novartis

Publisher's Note Springer Nature remains neutral with regard to jurisdictional claims in published maps and institutional affiliations. 